Preprints of the

Max Planck Institute for

Research on Collective Goods

Bonn 2014/7

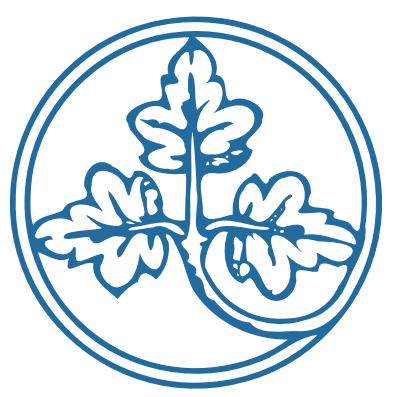

The values of ex-ante and ex-post communication in dictator games

Pascal Langenbach

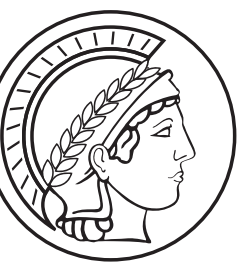




\title{
The values of ex-ante and ex-post communication in dictator games
}

\author{
Pascal Langenbach
}

Revised version: April 2016 


\title{
The values of ex-ante and ex-post communication in dictator games
}

\author{
Pascal Langenbach*
}

Revised version: April 2016

\begin{abstract}
In the dictator game, the recipient's opportunity to send a message to the dictator increases giving. This paper reports two experiments which study how the timing of messages affects dictators' decisions (experiment 1) and which value recipients attach to communication opportunities (experiment 2). The first experiment shows that the effect of communication on dictator giving is equally strong when the recipient can send a message before or after the dictator has decided. However, recipients in a second experiment reveal a strong preference for pre-decision messages: Their willingness to pay for pre-decision messages is higher than for post-decision messages.
\end{abstract}

Keywords: communication; altruism; inequality; dictator game

JEL Classification: D63, D64, C91, D83

\footnotetext{
* Max-Planck-Institute for Research on Collective Goods, Bonn, Germany. I am grateful to Christoph Engel, Susann Fiedler, Marco Kleine, Isabel Marcin, Pedro Robalo, Franziska Tausch, and Philipp Weinschenk for comments and discussion and to Nicolas Meier, Alexander Schneeberger, and Lars Freund for programming z-Tree and research assistance; Brian Cooper is thanked for translations and language help.
} 


\section{Introduction}

For many real-world actions, such as donations or favors, people do not expect material rewards. Thus, distributional fairness can be a powerful factor guiding human decision making (cf. Bolton and Ockenfels, 2000; Fehr and Schmidt, 1999, 2006). Yet, fairness concerns are often at odds with a decision maker's monetary self-interest, which arguably reduces their influence on behavior (cf. Andreoni and Rao, 2011). As a possible institutional intervention, decision making procedures could be designed in a way that strengthens the role of fairness in decision making. A promising procedural feature is to foster communication between the potential beneficiary and the decision maker (Andreoni and Rao, 2011; Hole, 2011). Communication prior to the decision is related to participation rights, which allow direct influencing of decision makers by providing information or opinions. But communication between the parties can also occur ex-post, i.e., when decisions are already made. Such feedback opportunities are frequent after many social interactions. They may serve as a way to express appraisal or grievance, e.g., by issuing a complaint. Hence, decision makers have to take responsibility for their actions.

This paper explores two "values" of communication in allocation decisions. First, I study how communication, and particularly the timing before or after a decision, affects its effectiveness to provide "fairer" outcomes. Secondly, I assess if and to what extent recipients of an allocation decision are willing to maintain a costly procedure that allows for one of the two communication options.

A widely-used experimental paradigm to investigate the conflict between selfish and other-regarding behavior is the dictator game (cf. Forsythe et al., 1994). I conducted two dictator game experiments where the recipients could or could not have the opportunity to send a message to the dictator, to study how the timing of communication affects generosity and how much the recipients value the opportunity to speak. In the dictator game, the recipient is totally dependent on the dictator's decision, whereas the dictator's decision is fully independent of the recipient's consent. Charness and Rabin (2005), however, report that dictators are responsive to the fact that recipients ask or do not ask for help prior to dictators' decisions. Yamamori et al. (2008) find that dictators react in their allocation to the recipients' requests.

A line of experiments has shown that the tension between fairness and self-interest can be resolved in favor of fairer decisions when recipients are allowed to communicate with 
the dictator. In a nutshell, usually "the squeaky wheel gets the grease": Pre-decision messages from the recipient to the dictator lead to more equal allocations in anonymous dictator games when recipients send a numerical request (Rankin, 2006), a written message (Mohlin and Johannesson, 2008), or both combined (Andreoni and Rao, 2011). ${ }^{1}$ Requests for more than $50 \%$ of the pie can have a negative effect (Rankin, 2006; Andreoni and Rao, 2011). Post-decision messages also affect the dictators' decisions: The previously announced opportunity for the recipient to write a message to the dictator after the decision increases average transfers (Ellingsen and Johannesson, 2008) and leads to less selfish decisions (Ellingsen and Johannesson, 2008; Xiao and Houser, 2009). In a complementary vein, Festre and Garrouste (2014) report higher generosity when a third-party observant gives verbal feedback to the dictator.

Several mechanisms have been put forward to explain why communication affects actions in the non-strategic dictator game setting (see Mohlin and Johannesson, 2008; Xiao and Houser, 2009; Andreoni and Rao, 2011 for the following discussion).

Communication could heighten the dictator's concern for the well-being of the recipient (Andreoni and Rao, 2011). This could be reached by reducing the social distance between players, which has been shown to affect dictators' decisions (Hoffman et al., 1996; Bohnet and Frey, 1999a, 1999b). Andreoni and Rao (2011) discuss two additional channels: First, communication could prevent "strategic ignorance" of the recipient's position in the attempt to keep a positive self-image. ${ }^{2}$ Dictators might be less able to ignore the recipients' point of view when they receive a pre-decision message. While predecision messages can immediately affect the dictators' decision making, post-decision messages cannot directly add information to the decision making process. Yet, dictators could anticipate the content of post-decision messages. ${ }^{3}$ Expected post-decision messages could thus also make it more difficult to remain ignorant of the recipients' concerns. Secondly, guilt aversion (Battigalli and Dufwenberg, 2007; Charness and Dufwenberg, 2006) could motivate the dictators to fulfill voiced requests if the dictators see requests

\footnotetext{
${ }^{1}$ Greiner et al. (2012) report positive effects of an audio-visual video message in a three-person dictator game.

${ }^{2}$ They relate their explanation to the findings of Dana et al. (2007) who show that some dictators do not want to know the consequences of their decisions for the recipient (see also Larson and Capra, 2009) and Grossman (2014) who reports that this only happens largely when the internal costs for remaining ignorant are not too high.

${ }^{3}$ Although they do not study post-decision messages, Andreoni and Rao report evidence that dictators are able to anticipate the content of later received pre-decision messages by the recipient and adjust their own prior message to the recipient to the anticipated content.
} 
as a proxy for the recipients' expectations. Likewise anticipated post-decision messages could prevent dictators from forming beliefs about the recipients' expectations that allow for selfish decisions (see Andreoni and Rao, 2011, for a discussion of guilt aversion and ex-ante communication).

Mohlin and Johannesson (2008) and Xiao and Houser (2009) use cognitive dissonance processes (Konow, 2000; introduced by Festinger, 1957) to explain the effects of predecision and post-decision messages in dictator games: (Anticipated) messages could provide the dictators with information about a normatively fair decision which could increase the dictators' internal costs to justify the payoff-maximizing allocation over the fair allocation. ${ }^{4}$

Finally, conditional on the content of the message, ex-post communication could function as a non-monetary negative or positive sanction which decision makers want to avert or gain (Xiao and Houser, 2009). ${ }^{5}$ If the dictators anticipate this use of post-decision messages, they could tailor their decisions to the expected message.

This previous research shows that ex-ante and ex-post communication is able to increase transfers from dictators to recipients. Experiment 1 also studies the effects of predecision and post-decision messages in a dictator game and allows to directly compare the relative effectiveness of both communication channels to increase the dictators' generosity. ${ }^{6}$

If messages from recipients to dictators can lead to fairer decisions, the recipients could make use of them to improve their outcomes. But do subjects understand the power of communication and value the opportunity to speak accordingly? This is particularly important because communication outside the laboratory is not always for free, but can come with costs. People have to make the effort to contact their counterpart, write a letter, make a call, etc. So even if communication is capable of delivering fairer outcomes, this is of little help if people misjudge its merits and fail to support communication procedures once costs are attached.

\footnotetext{
${ }^{4}$ Matthey and Regner (2011) use Konow's model to explain their data on "strategic ignorance".

${ }^{5}$ Similarly, Xiao and Houser (2005) report for an ultimatum game that receivers decline low offers by proposers less often if they can send a post-offer message and thus verbally "punish" the proposer.

${ }^{6}$ I am aware of one prior, yet so far unpublished, experiment presented at a workshop by Suleiman in 2011 which also studied pre-decision and post-decision messages in a single dictator game experiment. To the best of my knowledge, his experiment and results are not reported elsewhere. Experiment 1 was designed and conducted independently of this previous work.
} 
Recipients in dictator games frequently choose the option to have a mutual conversation with the dictator prior to the allocation decision, but do so less if this becomes costly (Frey and Bohnet, 1995). Further, recipients in the ultimatum game are willing to pay for pre-offer messages not only to the proposer, but also to the experimenter (Ong et al., 2013). Sending these messages in ultimate bargaining differs from sending messages in the dictator game: Messages towards proposers can be used to bargain, which does not hold for messages towards dictators because recipients in the dictator game have nothing to offer. Messages towards a third party cannot influence the proposer's decision making. Ong et al. (2013) argue that the bargaining setting in the ultimatum game already induces proposers to offer rather fair amounts. In contrast to this research, I study predecision and post-decision messages in the dictator game where recipients have no bargaining power vis-à-vis the dictator.

Subjects are also willing to pay for post-decision messages in a power-to-take game after finding out about their opponent's decision (Grosskopf and Lopez-Vargas, 2014). In the power-to-take game, the normative evaluation of the decision maker's action "to take" probably differs from the decision "not to share" in the dictator game. Subjects might, for example, feel more inclined to "punish" a decision maker who "stole" from them than a dictator who did not "give". Moreover, in my experiment, recipients indicate their willingness to pay when the dictator's decision is still uncertain. So subjects have to pay for an institution and their decisions are not driven by their experience in the specific interaction, i.e., the giving of the dictator.

Experiment 2 extends the existing research on costly communication by eliciting the recipients' willingness to pay for pre-decision and post-decision messages in the dictator game. Thus, I can compare the values recipients attach to either of the two communication channels.

In the first experiment, I replicate the finding that pre-decision and post-decision messages enhance generosity. Moreover, in the controlled setting of the dictator game, both channels seem to be equally effective. Dictators transfer similar amounts after having received a message beforehand or when they expect to receive a message after the decision. In the second experiment, I find that the recipients value the opportunity to express themselves to the dictator. They show a positive willingness to pay for predecision as well as for post-decision messages. Yet, their willingness to pay for predecision messages is higher. 
The paper is structured as follows: Section 2 describes the design and procedures of the two experiments. In section 3, I derive hypotheses for both experiments. Section 4 states the results. The paper ends with a brief conclusion in Section 5 .

\section{Experimental Design}

Two independent experiments with different subjects were conducted to study the research questions. I will first report the design of the first experiment, followed by the design of the second experiment.

\section{$2.1 \quad$ Experiment 1}

Upon arrival at the laboratory, participants were randomly assigned one of two roles, dictator or recipient, and then matched. After this, they were also randomly allocated to one of three treatments: baseline, pre-treatment, or post-treatment. This procedure and the three treatments were described in the instructions at the beginning of the experiment and were common knowledge for all participants. Therefore, participants were aware that different forms of communication existed, e.g., a dictator confronted with a recipient who had no opportunity to send a message knew that other recipients (in other treatments) could send messages. ${ }^{7}$

The baseline consisted of the standard dictator game. Dictators received an endowment of $100 \mathrm{MU}$ (monetary units) and could send any integer amount to the recipient. The transfer was the recipient's payoff; the dictator kept the remaining endowment. In the pre-treatment, the recipients sent a message containing a numerical request and a freeform written text to the dictator prior to the allocation. After the dictator had received the recipient's message, the dictator game was played as described. In the post-treatment, a numerical request (how much the recipient would have liked to receive) and a written message were sent after the dictator game had been played and the recipient had learned the dictator's allocation decision. The procedure was announced in the instructions, so that the dictator decided knowing that s/he would receive a message afterwards. In all

\footnotetext{
${ }^{7}$ This allows for potential comparisons between both experiments. As I will explain below, recipients in the second experiment could buy a communication option, thus participants in experiment 2 knew that some recipients could end up without the option to send a message. I keep this information constant between the two experiments by also informing participants in the first experiment about the different treatments in experiment 1.
} 
treatments, participants also answered standard post-experimental questionnaires (sociodemographics).

\subsection{Experiment 2}

The design of Experiment 2 mainly replicated the design of experiment 1, but additionally elicited the recipients' willingness to pay for pre-decision and post-decision messages by using a random price mechanism (cf. Becker et al., 1964). Subjects were again randomly assigned the role of dictator or recipient. Prior to the dictator game, recipients were given an endowment of $10 \mathrm{MU}$. Before being allocated to the different treatments, all recipients indicated how much of this endowment (with one decimal place) they would be willing to pay to send a pre-decision message if they were in the pre-treatment, and how much they would be willing to pay for a post-decision message if they were in the post-treatment. Only after this, were the recipients randomly allocated to the baseline, the pre-treatment, or the post-treatment. For participants in the pre-and post-treatment, the price of the communication option was determined by a randomly chosen number with one decimal place between 0 and 10 . If the recipient's stated amount equaled or exceeded the randomly chosen price, the recipient got the communication option available in the respective treatment. Recipients who received the communication option paid the randomly chosen price; the other recipients paid nothing. Therefore, their optimal strategy was to indicate an amount that reflects their maximum willingness to pay for communication.

For recipients who did not buy the communication option, the experimental design of the pre-and post-treatment equaled the baseline. Yet dictators learned if their matched recipient had the opportunity to purchase the communication option or not and whether s/he did so or not. Dictators did not learn how much the recipient was willing to pay or how much s/he actually paid. After the random price mechanism, the experiment proceeded as described for experiment 1. At the end of the experiment, the dictators reported their non-incentivized beliefs about how much the recipients had been willing to pay for either communication option.

\subsection{Procedures}

Experiments were computerized and conducted in the BonnEconLab at the University of Bonn using z-Tree (Fischbacher, 2007). 142 participants with a mean age of 24.4 years were recruited via ORSEE (Greiner, 2015) for the first experiment and 90 participants 
with a mean age of 23.4 years via HROOT (Bock et al., 2014) for the second experiment. ${ }^{8} 49.3$ (52.2) percent of the participants were female. Experimental instructions were distributed on paper prior to the experiments and read out aloud by the experimenter. Experiments lasted for around 45 minutes. MU were converted to Euro at a rate of MU $1=€ 0.1$ at the end of the experiment and paid out in cash. Participants earned a show-up fee of $€ 4$ in addition to their earnings from the dictator game.

Table 1

Treatment conditions (Communication Yes/No: Numbers of observations)

\begin{tabular}{cccc} 
& Experiment 1 (free) & \multicolumn{2}{c}{ Experiment 2 (costly) } \\
\hline Baseline & No: 23 & \multicolumn{3}{c}{ No: 15} \\
Pre-treatment & Yes: 24 & Yes: 4 & No: 11 \\
Post-treatment & Yes: 24 & Yes: 4 & No: 11 \\
\hline
\end{tabular}

\section{Hypotheses}

\subsection{Experiment 1}

Standard assumptions of self-centered and money-maximizing players do not provide any basis to predict treatment differences in this experiment: Independent of communication being present or not, dictators should not transfer any amount to recipients. Nevertheless, previous experiments constantly find that some dictators send money to the recipients (see Engel, 2011, for a meta-study). Moreover, according to the reported studies on communication from recipients to dictators, pre-decision and post-decision messages induce dictators to give more to recipients. Therefore, I also expect that in the pre-treatment and the post-treatment dictators transfer higher amounts to the recipients than in the baseline (H1).

While the dictators learn the content of pre-decision messages before they make their decision, they do not yet know the content of post-decision messages when they decide. The effects of ex-post communication on decision making largely depend on the

\footnotetext{
${ }^{8}$ Experiment 1 was conducted in late August 2013, and so were sessions to study recipients' willingness to pay for communication. The data of the latter sessions, still included in the initial version of this preprint, had to be discarded due to a later discovered error. I ran corrected sessions of experiment 2 in September 2015. Only these additional sessions are reported here.
} 
anticipation of messages. I conjecture that it is easier for the dictators to dismiss the anticipated content of post-decision messages than the content of already received predecision messages. This does not mean that post-decision messages will have no effect. As Xiao and Houser (2009) elaborate, dictators can hardly choose to ignore the content of post-decision messages, e.g., by choosing not to read them, without acknowledging that they might contain an expression of discontent with a certain allocation. Similarly, Andreoni and Rao (2011) argue that the fact that dictators consider the recipients' concerns already increases transfers. However, since the anticipation of communication leaves some room for the dictators to deceive themselves about what the recipients might write, anticipated communication could be less effective in reducing selfish behavior than already experienced communication. Consequently, I predict that transfers from dictator to recipient will be higher in the pre-treatment than in the post-treatment $(\mathrm{H} 2)$.

\subsection{Experiment 2}

The second experiment mainly studies the recipients' willingness to pay for messages. If the recipients anticipate the effects of pre-decision and post-decision messages on dictator giving, they should be willing to buy the communication option. This should result in a positive willingness to pay for pre-decision messages and post-decision messages. As predicted in $\mathrm{H} 2$, the recipients might think that the effects of pre-decision messages outweigh the effects of post-decision messages because the latter depend on anticipation. The post-decision mechanisms might be more difficult for the recipients to imagine, which supports the hypothesis that the recipients will show a higher willingness to pay for pre-decision messages than for post-decision messages (H3).

\section{Results}

First, I report the effects of pre-decision and post-decision messages on dictator decisions in experiment 1. Mean transfers from dictator to recipient are higher in the pretreatment (26.79 MU) and in the post-treatment (28.83 MU) than in the baseline (17.43 MU) (Figure 1). Without communication, $48 \%$ of dictators give nothing to the recipient. This number drops to $13 \%$ with ex-ante and $21 \%$ with ex-post communication. Differences in transfers between the baseline and the communication treatments are significant according to a Mann-Whitney test (baseline vs. pre-treatment: $|\mathrm{z}|=2.122$, 
$\mathrm{p}=0.0338$; baseline vs. post-treatment: $\left.|\mathrm{z}|=2.246, \mathrm{p}=0.0247^{9}\right)$. In line with previous experimental research, I find a fairness-increasing effect of pre-decision and post-decision messages on dictator giving and therefore support for $\mathrm{H} 1$.

When recipients can send a message, they state nearly identical numerical requests in the pre-treatment (40.29 MU) and the post-treatment (39.79 MU; Mann-Whitney: $|\mathrm{z}|=0.011, \mathrm{p}=0.9912)$. Comparing the transfers between both communication treatments, I cannot reject the null hypothesis that dictators share the same amount (MannWhitney: $|z|=0.26, p=0.7946)$. I find no differences in the effects on dictator giving between actually experienced messages and anticipated messages, and hence no support for $\mathrm{H} 2$.

Transfers in dictator games are subject to gender effects: Female dictators give more to recipients (see Engel, 2011). I control for the effect of the dictators' gender on transfers using regression analyses (Table 2 and 3). I use Tobit regressions because data on transfers in dictator games is censored at the zero giving level. Yet, as shown by Bardsley (2008), (some) dictators also take money if possible. Tobit regressions control for censoring. The results hold if a female dummy is entered in the regression equation: The coefficients of the treatment dummies remain significant when giving between the baseline and the communication treatments is compared (model 2 in Table 2) and insignificant when I compare giving in the pre- and post-treatment (model 2 in Table 3).

Result 1: Transfers are higher in pre- and post-treatment than in the baseline.

Result 2: Pre-decision and post-decision messages are equally effective in inducing dictators to give more.

A possible explanation as to why pre-decision and post-decision messages have similar effects on giving could be that the fairness norm of an equal split and thus the content of messages in dictator games are rather obvious (cf. Andreoni and Rao, 2011). Therefore, when dictators think about the post-decision message, they might not reach a different conclusion about a fair allocation as when they actually receive a pre-decision message. Interestingly, though, giving in the post-treatment is higher than in the baseline, even though baseline dictators also knew that recipients in the communication treatments could send messages. One could have expected that baseline dictators also speculated about the content of communication in the other treatments. The comparison between

\footnotetext{
${ }^{9}$ All reported tests are two-sided.
} 
baseline and post-treatment shows that the expectation that one will receive a message in a specific relationship has a strong effect. Only the abstract knowledge that communication takes place in a similar setting does not lead to comparable levels of giving. This corroborates the general interpretation of the effects of verbal feedback by Ellingsen and Johannesson (2008), who state that " $[\mathrm{k}]$ nowing that someone is justifiably angry does not arouse the same level of shame as facing the angry person, or even having to read angry messages."

Figure 1: Average transfers in experiment 1 by treatment (yellow lines indicate $95 \% \mathrm{CI}$ )

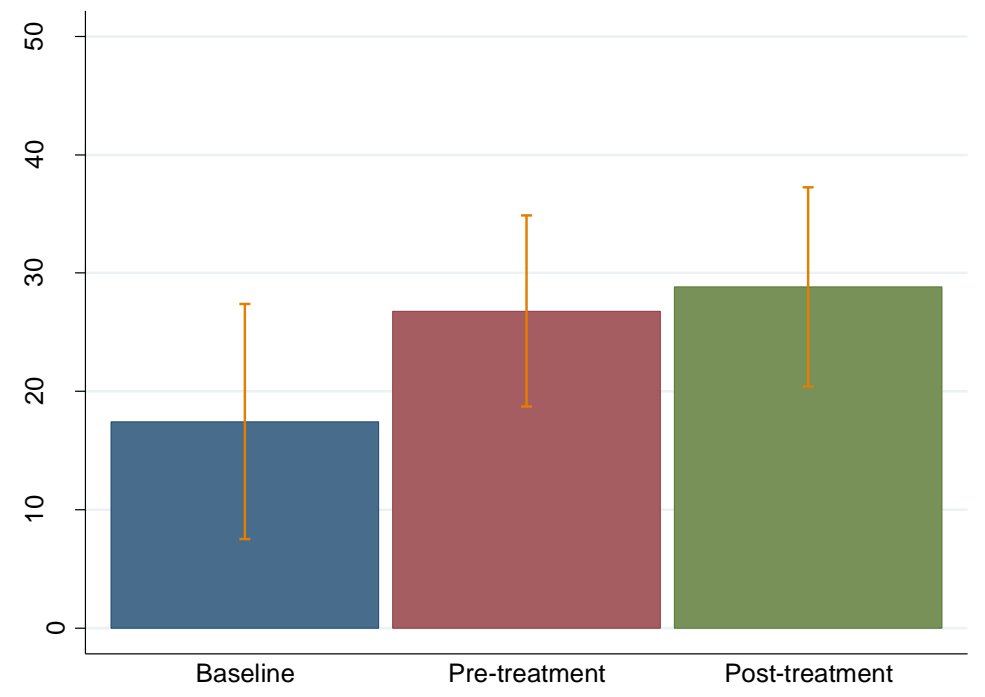

Table 2: Treatment effects on dictator giving in experiment 1 (baseline versus communication treatments) - Tobit regressions

\begin{tabular}{|c|c|c|}
\hline & Model 1 & Model 2 \\
\hline Pre-treatment & $16.24 * *(8.08)$ & $17.95 * *(7.88)$ \\
\hline Post-treatment & $17.15 * *(8.09)$ & $18.30 * *(7.86)$ \\
\hline Female & & $14.23 * *(6.31)$ \\
\hline Constant & $8.80 \quad(6.03)$ & $(7.07)$ \\
\hline$N$ & 71 & 71 \\
\hline left-censored & 19 & 19 \\
\hline
\end{tabular}

Notes: Pre-treatment and Post-treatment dummies equal one for participants in the respective treatment and zero otherwise. Female dummy equals 1 for female participants;

Standard errors in parentheses; ** significance level 0.05 
Table 3: Treatment effects on dictator game transfers in experiment 1 (pre-treatment versus post-treatment) - Tobit regressions

\begin{tabular}{ccc}
\hline & Model 1 & Model 2 \\
\hline Post-treatment & $1.15(6.57)$ & $0.72(6.35)$ \\
Female & & $10.99 *(6.36)$ \\
Constant & $25.39 * * *(4.64)$ & $20.39 * * *(5.36)$ \\
\hline$N$ & 48 & 48 \\
left-censored & 8 & 8
\end{tabular}

Notes: Post-treatment dummy equals one for participants in the post-treatment and zero for participants in the pre-treatment. Female dummy equals 1 for female participants;

Standard errors in parentheses; *, *** significance levels $0.1,0.01$

I now turn to the analysis of experiment 2, where I elicited the subjects' willingness to pay for communication. I collected 45 observations for the two communication options. While 40 recipients indicated a positive willingness to pay for pre-decision messages, only 27 did so for post-decision messages. Of the 45 recipients, 28 showed a higher willingness to pay for pre-decision messages than for post-decision messages, and only 5 preferred post-decision messages; 12 recipients stated equal amounts. The average bid was $3.99 \mathrm{MU}$ for pre-decision messages and $2.42 \mathrm{MU}$ for post-decision messages. The difference in the willingness to pay is statistically significant according to a Wilcoxon signed-ranks test $(|\mathrm{z}|=3.879, \mathrm{p}=0.0001)$. Conditional on a positive willingness to pay for a pre-decision message and a post-decision message, the recipients' bids for pre-decision messages are also significantly higher than the bids for post-decision messages $(\mathrm{N}=26$; pre-decision: $5.05 \mathrm{MU}$, post-decision: 4.12 MU; Wilcoxon signed-ranks test: $|\mathrm{z}|=2.262$, $\mathrm{p}=0.0237) .{ }^{10}$ Recipients have a positive willingness to pay for both forms of communication; still, as stated in H3, they value pre-decision messages more than postdecision messages.

Result 3: Recipients show a higher willingness to pay for pre-decision messages than for post-decision messages.

\footnotetext{
${ }^{10}$ Only one subject showed a positive willingness to pay for the post-decision message and none for the pre-decision message.
} 
If one compares the recipients' willingness to pay for messages with the effects of messages on dictator giving in experiment 1, the recipients seem to bid rather low amounts. In the first experiment, communication increased transfers on average by 9.36 $\mathrm{MU}$ in the pre-treatment and by $11.4 \mathrm{MU}$ in the post-treatment. I conjecture that costly messages also have positive effects on the dictators' decisions. ${ }^{11}$ Recipients who pay for messages signal that talking to the dictator is important to them, which could arguably make communication even more effective. Thus, in order to maximize their payoff, recipients in experiment 2 should have bid higher amounts for communication. It seems that they are not fully aware of the power of communication on the dictators' decisions.

Finally, I report an additional explorative finding. I study the differences between the baselines of the two experiments. No communication took place in either baseline. If the dictators mainly want to reduce inequality, they should give more to recipients with a lower endowment. Inequality is greater in experiment 1 than in experiment 2 because the recipients in the baseline of the first experiment had no initial endowment, whereas the recipients in the baseline of the second experiment received an endowment of $10 \mathrm{MU}$ and did not spend it. Previous studies found that the size of the recipients' endowment predicts dictator giving (see Engel, 2011); thus, one would expect that dictators allocate more to recipients in the baseline of experiment 1 than to recipients in the baseline of experiment 2. If I compare the baselines of both experiments, transfers are even higher in the baseline of the second experiment than in the baseline of the first experiment; the difference being (marginally) statistically significant (17.43 MU/25.73 MU; Mann Whitney test: $|\mathrm{z}|=1.825, \mathrm{p}=0.0681$ ). However, using a Tobit regression, the experiment dummy only turns out to be (marginally) significant if I additionally control for the dictators' gender (Table 4). Even though the robustness of the result of higher giving in the baseline of experiment 2 can hence be questioned, I conclude that transfers in the baseline of the second experiment are not - as expected - lower than those in the baseline of the first experiment. This indicates that the initial endowment of the recipients does not dominate the transfers in the baseline of the second experiment.

\footnotetext{
${ }^{11}$ The low number of observations in which communication actually took place in the second experiment $(\mathrm{N}=4$ for each communication treatment) excludes a meaningful statistical analysis of the effects of costly messages.
} 
Table 4: Baseline transfers in both experiments -

Tobit regressions

\begin{tabular}{ccc}
\hline & Model 1 & Model 2 \\
\hline Experiment & $15.81(9.99)$ & $16.54 *(9.63)$ \\
Female & & $19.66^{*}(9.72)$ \\
Constant & $7.79(6.74)$ & $-3.64(9.09)$ \\
& & \\
\hline$N$ & 38 & 38 \\
Left-censored & 13 & 13 \\
\hline
\end{tabular}

Notes: Experiment equals one for the second experiment and zero for the first.

The Female dummy equals one for female participants.

Standard errors in parentheses. * significance level 0.1

\section{Conclusions}

If the recipient sends a message, the dictator gives more. The first experiment replicated the fairness-enhancing effects of ex-ante and ex-post communication in a setting where dictators were aware that other states of the world existed, i.e., the dictators in the communication treatments knew that the dictators in the baseline did not receive messages. Surprisingly, I find no difference between the effects of pre-decision and postdecision messages. In the dictator game, the anticipation of messages seems to work as well as a "real experience".

The second experiment paints a more nuanced picture. The recipients value both communication options differently. They show a higher willingness to pay for predecision messages than for post-decision messages. Overall, it seems that recipients underestimate the power of communication. Given the positive effects of anticipated and experienced messages on generosity, they should have bid more to buy messages in the second experiment.

Hence, communication in decision procedures poses a subtle instrument to increase fairness in non-strategic decision making even if communication is only anticipated. That said, recipients are not equally willing to invest to maintain such procedures; they prefer to be heard before decisions are taken. 


\section{References}

Andreoni, J., Rao, J. M. (2011). The power of asking: How communication affects selfishness, empathy, and altruism. Journal of Public Economics 95 (7-8), 513-520.

Bardsley, N. (2008). Dictator game giving: altruism or artefact?. Experimental Economics 11 (2), 122-133.

Battigalli, P., Dufwenberg, M. (2007). Guilt in games. The American Economic Review 97 (2), 170-176.

Becker, G. M., DeGroot, M. H., Marschak, J. (1964). Measuring utility by a single-response sequential method. Behavioral Science 9 (3), 226-232.

Bock, O., Baetge, I., Nicklisch, A. (2014). hroot: Hamburg registration and organization online tool. European Economic Review 71, 117-120.

Bohnet, I., Frey, B. S. (1999a). The sound of silence in prisoner's dilemma and dictator games. Journal of Economic Behavior 83 Organization 38 (1), 43-57.

Bohnet, I., Frey, B.S. (1999b). Social distance and other-regarding behavior in dictator games: Comment. The American Economic Review 89 (1), 335-339.

Bolton, G.E., Ockenfels, A. (2000). ERC: A theory of equity, reciprocity, and competition. The American Economic Review 90 (1), 166-193.

Charness, G., Dufwenberg, M. (2006). Promises and partnership. Econometrica 74 (6), 1579-1601.

Charness, G., Rabin, M. (2005). Expressed preferences and behavior in experimental games. Games and Economic Behavior 53 (2), 151-169.

Dana, J., Weber, R. A., \& Kuang, J. X. (2007). Exploiting moral wiggle room: experiments demonstrating an illusory preference for fairness. Economic Theory, 33 (1), 67-80

Ellingsen, T., Johannesson, M. (2008). Anticipated verbal feedback induces altruistic behavior. Evolution and Human Behavior 29 (2), 100-105.

Engel, C. (2011). Dictator games: A meta study. Experimental Economics 14 (4), 583-610.

Fehr, E., Schmidt, K.M. (1999). A theory of fairness, competition, and cooperation. The Quarterly Journal of Economics 114 (3), 817-868.

Fehr, E., Schmidt, K. M. (2006). The economics of fairness, reciprocity and altruism - experimental evidence and new theories. Handbook of the economics of giving, altruism and reciprocity 1, 615-691.

Festinger, L. (1957). A theory of cognitive dissonance.

Festré, A., Garrouste, P. (2014). Somebody may scold you! A dictator experiment. Journal of Economic Psychology 45, 141-153.

Fischbacher, U. (2007). z-Tree: Zurich toolbox for ready-made economic experiments. Experimental Economics 10 (2), 171-178.

Forsythe, R., Horowitz, J.L., Savin, N.E., Sefton, M. (1994). Fairness in simple bargaining experiments. Games and Economic Behavior 6 (3), 347-369. 
Frey, B.S., Bohnet, I. (1995). Institutions affect fairness: Experimental investigations. Journal of Institutional and Theoretical Economics 151 (2), 286-303.

Greiner, B., Güth, W., Ro'I, Z. (2012). Social communication and discrimination: A video experiment. Experimental Economics 15 (3), 398-417.

Greiner, B. (2015). Subject pool recruitment procedures: organizing experiments with ORSEE. Journal of the Economic Science Association 1 (1), 114-125.

Grosskopf, B., Lopez-Vargas, K. (2014). On the demand for expressing emotions. Working Paper.

Grossman, Z. (2014). Strategic ignorance and the robustness of social preferences. Management Science 60 (11), 2659-2665.

Hoffman, E., McCabe, K., Smith, V. L. (1996). Social distance and other regarding behavior in dictator games. The American Economic Review 86 (3), 653-660.

Hole, A. D. (2011). Communication and fair distribution. Rationality and Society, 23 (2), 234-264.

Konow, J. (2000). Fair shares: Accountability and cognitive dissonance in allocation decisions. The American Economic Review 90 (4), 1072-1091.

Larson, T., Capra, C. M. (2009). Exploiting moral wiggle room: Illusory preference for fairness? A Comment. Judgment and Decision Making 4(6), 467-474.

Matthey, A., Regner, T. (2011). Do I really want to know? A cognitive-dissonance based explanation of other regarding behavior. Games 2 (1), 114-135.

Mohlin, E., Johannesson, M. (2008). Communication: Content or relationship? Journal of Economic Behavior \&3 Organization 65 (3-4), 409-419.

Ong, Q., Riyanto, Y. E., Theseira, W. E., Sheffrin, S. M. (2013). When words speak louder than actions: The self-image signaling role of voice in the ultimatum game. Working Paper.

Rankin, F.W. (2006). Requests and social distance in dictator games. Journal of Economic Behavior 83 Organization 60 (1), 27-36.

Suleiman, R. (2011). Effects of ex ante and ex post communication on behavior in simple bargaining games. Presentation slides from the workshop: Experimental approaches in conflict research, January 3-4, 2011, Jerusalem.

Xiao, E., Houser, D. (2005). Emotion expression in human punishment behavior. Proceedings of the National Academy of Sciences 102 (20), 7398-7401.

Xiao, E., Houser, D. (2009). Avoiding the sharp tongue: Anticipated written messages promote fair economic exchange. Journal of Economic Psychology 30 (3), 393-404.

Yamamori, T., Kato, K., Kawagoe, T., Matsui, A. (2008). Voice matters in a dictator game. Experimental Economics 11 (4), 336-343. 


\section{Appendix - Experimental Instructions}

Experiments were conducted in German. The instructions have been translated into English.

\section{Instructions Experiment 1}

\section{Information about the Experiment}

Thank you for participating in today's experiment. Please read the following information carefully. The experiment will be conducted anonymously, i.e., nobody is told with which of the other participants he or she has interacted. The experiment evaluation is also anonymous. Depending on your decisions and the decisions made by the other participants, you can earn money in this experiment. In this experiment, we shall speak not of Euro, but of Taler. After the experiment, each Taler is converted into Euro at a rate of 1 Taler $=0.10 €$. In addition, every participant gets a fee of $4 €$ for showing up today. Your earnings are paid to you in cash at the end of the experiment.

There are two roles in this experiment: $\mathbf{A}$ and $\mathbf{B}$. Your role is randomly assigned to you at the beginning of the experiment. One role $A$ participant and one role $B$ participant are assigned to each other. You will not be told who the other participant is.

Participant $A$ is given an endowment of 100 Taler at the beginning of the experiment. Participant $A$ decides how many of these 100 Taler he or she wishes to send to participant B. The Taler that are sent are credited to Participant B's account. The Taler that have not been sent remain with Participant A.

There are three different situations in which this decision is made: Situation 1, Situation 2, or Situation 3. Each situation can occur with a probability of $1 / 3$. Hence, whether you are in situation 1 , situation 2 , or in situation 3 is equally probable.

The three situations are now described in detail:

\section{Situation 1:}

In situation 1, Participant $B$ has the opportunity to send Participant $\boldsymbol{A}$ a message, before the latter makes a decision on the distribution of the 100 Taler. For this purpose, Participant $B$ is shown a description field on the screen, where the message to Participant $A$ can be entered. In addition, Participant $B$ enters the minimum sum he or she would like to receive. This message and the minimum sum are then shown to Participant $A$, also on the screen. Following that, Participant $A$ makes a decision on how to divide the 100 Taler among the two participants. This distribution is shown to Participant $B$. In situation 1, the experiment ends here.

\section{Situation 2:}

In situation 2, Participant $B$ has the opportunity to send Participant $\boldsymbol{A}$ a message, after the latter has made a decision on the distribution. First, Participant $A$ makes a decision on how to divide the 100 Taler among both participants. This distribution is shown to Participant 
B. Participant $B$ is then shown a description field on the screen, where the message to Participant $A$ can be entered. In addition, Participant $B$ enters the minimum sum he or she would have liked to receive. This message and the minimum sum are then shown to Participant $A$, also on the screen. In situation 2 , the experiment ends here.

\section{Situation 3:}

In situation 3, Participant $A$ makes a decision on how to divide the 100 Taler among both participants. There is no possibility to send any message. The decision made by Participant $A$ is shown to Participant $B$ on the screen. In situation 3, the experiment ends here.

\section{Instructions Experiment 2}

\section{Information about the Experiment}

Thank you for participating in today's experiment. Please read the following information carefully. The experiment will be conducted anonymously, i.e., nobody is told with which of the other participants he or she has interacted. The experiment evaluation is also anonymous. Depending on your decisions and the decisions made by the other participants, you can earn money in this experiment. In this experiment, we shall speak not of Euro, but of Taler. After the experiment, each Taler is converted into Euro at a rate of 1 Taler $=\mathbf{0 . 1 0} €$. In addition, every participant gets a fee of $4 €$ for showing up today. Your earnings are paid to you in cash at the end of the experiment.

There are two roles in this experiment: $\mathbf{A}$ and $\mathbf{B}$. Your role is randomly assigned to you at the beginning of the experiment. One role $A$ participant and one role $B$ participant are assigned to each other. You will not be told who the other participant is.

Participant $A$ is given an endowment of 100 Taler at the beginning of the experiment. Participant $A$ decides how many of these 100 Taler he or she wishes to send to participant $B$. The Taler that are sent are credited to Participant B's account. The Taler that have not been sent remain with Participant $A$.

There are three different situations in which this decision is made: Situation 1, Situation 2, or Situation 3. Each situation can occur with a probability of $1 / 3$. Hence, whether you are in situation 1 , situation 2 , or in situation 3 is equally probable.

- In Situation 1, Participant $B$ may purchase the opportunity to send Participant $A$ a message before Participant $A$ divides the 100 Taler.

- In Situation 2, Participant $B$ may purchase the opportunity to send Participant $A$ a message after Participant $A$ has divided the 100 Taler.

- In Situation 3, Participant B may not purchase any opportunity to send Participant $A$ a message. 
The following lines explain how participant $B$, at the beginning of the experiment, can purchase the opportunity to send a message:

Participant $B$ receives an endowment of $\mathbf{1 0 . 0}$ Taler. Participant $B$ is not yet told in which situation $(1,2$, or 3$)$ he or she is. Participant $B$ therefore takes two decisions:

- The first decision is taken as though Participant B is in situation 1. In other words, Participant $B$ states how many Taler of the endowment he or she is willing to pay at the most in order to send Participant $A$ a message, before the latter divides the 100 Taler.

- The second decision is taken as though Participant $B$ is in situation 2. In other words, Participant $B$ states how many Taler of the endowment he or she is willing to pay at the most in order to send Participant $A$ a message, after the latter has divided the 100 Taler.

Since there is no opportunity in situation 3 to send a message, Participant $B$ cannot name a sum in this case.

Please note, if you are Participant $B$, that you do not yet know, at the time of stating how many Taler you are prepared to pay, in which situation $(1,2$, or 3) you are. Please take both of your decisions carefully, as both can become payoff-relevant with the same probability. You may use up to 10.0 Taler for each of the two decisions.

After Participant $B$ has made both decisions, he/she is told in which situation $(1,2$, or 3$)$ he/she is.

In situations 1 and $\mathbf{2}$, the actual price for a message to Participant $A$ is calculated afterwards: The computer randomly determines a price between $\mathbf{0 . 0}$ and $\mathbf{1 0 . 0}$ Taler. Every price between 0.0 and 10.0 Taler is equally probable. The price is individually calculated for each Participant B, independently of the prices for the other Participants $B$.

In situation 1, the actual price is compared to the sum Participant $B$ is prepared to pay in order to send Participant $A$ a message before the division decision. In situation 2 , the actual price is compared to the sum Participant $B$ is prepared to pay in order to send Participant $A$ a message after the division decision.

- If the actual price is lower than the sum Participant $B$ is prepared to pay, or if the price is the same as that sum, then Participant $B$ has purchased the opportunity to send Participant $A$ a message. The price for sending the message is subtracted from Participant B's endowment.

- If the price is higher than the sum Participant $B$ is prepared to pay to send a message, then Participant $B$ has not purchased the opportunity to send Participant $A$ a message. In this case, nothing is subtracted from Participant B's endowment.

Since no opportunity to send a message can be purchased in situation 3, no price is calculated in this situation, and nothing is subtracted from Participant B's endowment. 
Participant $A$ is told in which situation he or she is. In situations 1 and 2 , in addition, Participant $A$ is told whether Participant $B$ has purchased the opportunity to send a message. However, Participant $A$ does not find out how high the price was for the message, nor how many Taler Participant $B$ was prepared to pay.

The three situations are now described in detail:

Situations 1 and 2 are described in case Participant $\boldsymbol{B}$ has purchased the opportunity to send a message:

\section{Situation 1:}

In situation 1, participant $B$ has the opportunity to send Participant $\boldsymbol{A}$ a message, before the latter makes a decision on the distribution of the 100 Taler. For this purpose, Participant $B$ is shown a description field on the screen, where the message to Participant $A$ can be entered. In addition, Participant $B$ enters the minimum sum he or she would like to receive. This message and the minimum sum are then shown to Participant $A$, also on the screen. Following that, Participant $A$ makes a decision on how to divide the 100 Taler among the two participants. This distribution is shown to Participant B. In situation 1, the experiment ends here.

\section{Situation 2:}

In situation 2, Participant $B$ has the opportunity to send Participant $\boldsymbol{A}$ a message, after the latter has made a decision on the distribution. First, Participant $A$ makes a decision on how to divide the 100 Taler among both participants. This distribution is shown to Participant $B$. Participant $B$ is then shown a description field on the screen, where the message to Participant $A$ can be entered. In addition, Participant $B$ enters the minimum sum he or she would have liked to receive. This message and the minimum sum are then shown to Participant $A$, also on the screen. In situation 2, the experiment ends here.

In case Participant $B$ has not purchased the opportunity to send a message, situations 1 and 2 are played just like situation 3 .

\section{Situation 3:}

In situation 3 (or in situations 1 and 2, if no opportunity to send a message was purchased), Participant $A$ makes a decision on how to divide the 100 Taler among both participants. There is no possibility to send any message. The decision made by Participant $A$ is shown to Participant $B$ on the screen. In situation 3 , the experiment ends here. 